PROCEEDINGS OF THE

AMERICAN MATHEMATICAL SOCIETY

Volume 136, Number 11, November 2008, Pages 3803-3807

S 0002-9939(08)09422-7

Article electronically published on May 22, 2008

\title{
THE COHOMOLOGY OF THE HEISENBERG LIE ALGEBRAS OVER FIELDS OF FINITE CHARACTERISTIC
}

\author{
GRANT CAIRNS AND SEBASTIAN JAMBOR
}

(Communicated by Dan M. Barbasch)

\begin{abstract}
We give explicit formulas for the cohomology of the Heisenberg Lie algebras over fields of finite characteristic. We use this to show that in characteristic two, unlike all other cases, the Betti numbers are unimodal.
\end{abstract}

The Heisenberg Lie algebra is the algebra $\mathfrak{h}_{m}$ with basis $\left\{x_{1}, \ldots, x_{m}, y_{1}, \ldots\right.$, $\left.y_{m}, z\right\}$ and nonzero relations $\left[x_{i}, y_{i}\right]=z, 1 \leq i \leq m$. The cohomology (with trivial coefficients) $H^{*}\left(\mathfrak{h}_{m}\right)$ was one of the first explicit computations of the cohomology of a family of nilpotent Lie algebras. Louis Santharoubane [4] showed that over fields of characteristic zero, the Betti numbers are:

$$
\operatorname{dim} H^{n}\left(\mathfrak{h}_{m}\right)=\left(\begin{array}{c}
2 m \\
n
\end{array}\right)-\left(\begin{array}{c}
2 m \\
n-2
\end{array}\right),
$$

for all $n \leq m$. Over fields of prime characteristic, the differential has larger kernel, and so one expects "more" cohomology. Recently, Emil Sköldberg [5] used algebraic Morse theory to compute the Poincaré polynomial $S_{m}(t)=\sum_{n} \operatorname{dim} H^{n}\left(\mathfrak{h}_{m}\right) t^{n}$ of the Heisenberg Lie algebra $\mathfrak{h}_{m}$ over fields of characteristic two. He obtained

$$
S_{m}(t)=\frac{\left(1+t^{3}\right)(1+t)^{2 m}+\left(t+t^{2}\right)(2 t)^{m}}{1+t^{2}} .
$$

In this paper we extend Sköldberg's result to arbitrary characteristic by directly computing the Betti numbers.

Theorem. Over fields of characteristic $p$, one has

$$
\operatorname{dim} H^{n}\left(\mathfrak{h}_{m}\right)=\left(\begin{array}{c}
2 m \\
n
\end{array}\right)-\left(\begin{array}{c}
2 m \\
n-2
\end{array}\right)+\sum_{i=1}^{\left\lfloor\frac{n+1}{2 p}\right\rfloor}\left(\begin{array}{c}
2 m+1 \\
n-2 i p+1
\end{array}\right)-\sum_{i=1}^{\left\lfloor\frac{n-1}{2 p}\right\rfloor}\left(\begin{array}{c}
2 m+1 \\
n-2 i p-1
\end{array}\right),
$$

for all $i \leq m$.

In particular, we have:

Corollary 1. In characteristic two,

$$
\operatorname{dim} H^{n}\left(\mathfrak{h}_{m}\right)=\sum_{i=0}^{\left\lfloor\frac{n}{2}\right\rfloor}(-1)^{i}\left(\begin{array}{c}
2 m \\
n-2 i
\end{array}\right)+\sum_{i=0}^{\left\lfloor\frac{n-3}{2}\right\rfloor}(-1)^{i}\left(\begin{array}{c}
2 m \\
n-3-2 i
\end{array}\right),
$$

for all $i \leq m$.

Received by the editors November 6, 2006, and, in revised form, September 23, 2007.

2000 Mathematics Subject Classification. Primary 17B55, 17 B56.

(C)2008 American Mathematical Society 
The characteristic two case of the theorem is equivalent to Sköldberg's formula, as can be seen by expanding the latter, applying the binomial expansion to the factor $(1+t)^{2 m}$, and using the expansion $\frac{1}{1+t^{2}}=\sum_{n=0}(-1)^{n} t^{2 n}$. Recall that the Betti numbers $\operatorname{dim} H^{n}(L)$ of a unimodular Lie algebra $L$ satisfy Poincaré duality (that is, $\operatorname{dim} H^{n}(L)=\operatorname{dim} H^{\operatorname{dim} L-n}(L)$ ), and they are said to be unimodal if they increase with $n$ for $1 \leq n \leq \frac{1}{2} \operatorname{dim} L$ and then (consequently) decrease for $\frac{1}{2} \operatorname{dim} L \leq n \leq \operatorname{dim} L$. One of the utilities of Santharoubane's result was that, over fields of characteristic zero, it provided examples of nilpotent Lie algebras whose Betti numbers are not unimodal; see 2] and 1]. Other nilpotent algebras with non-unimodal Betti numbers were given in 3. In characteristic $p>2$, the above theorem gives $\operatorname{dim} H^{3}\left(\mathfrak{h}_{4}\right)=\left(\begin{array}{l}8 \\ 3\end{array}\right)-\left(\begin{array}{l}8 \\ 1\end{array}\right)=48$, while $\operatorname{dim} H^{4}\left(\mathfrak{h}_{4}\right)=\left(\begin{array}{l}8 \\ 4\end{array}\right)-\left(\begin{array}{l}8 \\ 2\end{array}\right)=42$, exactly as in the characteristic zero case. However, we have:

Corollary 2. In characteristic two, for all $m \geq 1$, the Betti numbers of $\mathfrak{h}_{m}$ are (strictly) unimodal; that is, $\operatorname{dim} H^{n}\left(\mathfrak{h}_{m}\right)>\operatorname{dim} H^{n-1}\left(\mathfrak{h}_{m}\right)$, for all $1 \leq n \leq m$.

This raises an obvious question: over fields of characteristic two, do all nilpotent Lie algebras have unimodal Betti numbers?

Proof of the theorem. Consider the differential $d$ in the exterior algebra $\Lambda \mathfrak{h}_{m}^{*}$ over the dual vector space $\mathfrak{h}_{m}^{*}$, where by definition $d: \mathfrak{h}_{m}^{1} \rightarrow \Lambda \mathfrak{h}_{m}^{2}$ is the dual of the Lie bracket map. For each $n$, let $Z^{n}$ denote the kernel of $d: \Lambda^{n} \mathfrak{h}_{m}^{*} \rightarrow \Lambda^{n+1} \mathfrak{h}_{m}^{*}$. Since $H^{*}\left(\mathfrak{h}_{m}\right)=\operatorname{kernel}(d) /$ image $(d)$, one has

$$
\operatorname{dim} H^{n}\left(\mathfrak{h}_{m}\right)=\operatorname{dim} Z^{n}+\operatorname{dim} Z^{n-1}-\left(\begin{array}{c}
2 m+1 \\
n-1
\end{array}\right) .
$$

We claim that for $n \leq m$,

$$
\operatorname{dim} Z^{n}=\left(\begin{array}{c}
2 m \\
n
\end{array}\right)+\sum_{i=1}^{\left\lfloor\frac{n+1}{2 p}\right\rfloor}\left(\begin{array}{c}
2 m \\
n-2 i p+1
\end{array}\right)-\sum_{i=1}^{\left\lfloor\frac{n-1}{2 p}\right\rfloor}\left(\begin{array}{c}
2 m \\
n-2 i p-1
\end{array}\right)
$$

Note that using the binomial formula,

$$
\left(\begin{array}{l}
k \\
i
\end{array}\right)=\left(\begin{array}{c}
k-1 \\
i
\end{array}\right)+\left(\begin{array}{c}
k-1 \\
i-1
\end{array}\right),
$$

(11) and (2) give

$$
\begin{aligned}
\operatorname{dim} H^{n}\left(\mathfrak{h}_{m}\right)= & \left(\begin{array}{c}
2 m \\
n
\end{array}\right)-\left(\begin{array}{c}
2 m \\
n-2
\end{array}\right)+\sum_{i=1}^{\left\lfloor\frac{n+1}{2 p}\right\rfloor}\left(\begin{array}{c}
2 m \\
n-2 i p+1
\end{array}\right)-\sum_{i=1}^{\left\lfloor\frac{n-1}{2 p}\right\rfloor}\left(\begin{array}{c}
2 m \\
n-2 i p-1
\end{array}\right) \\
& +\sum_{i=1}^{\left\lfloor\frac{n}{2 p}\right\rfloor}\left(\begin{array}{c}
2 m \\
n-2 i p
\end{array}\right)-\sum_{i=1}^{\left\lfloor\frac{n-2}{2 p}\right\rfloor}\left(\begin{array}{c}
2 m \\
n-2 i p-2
\end{array}\right) \\
= & \left(\begin{array}{c}
2 m \\
n
\end{array}\right)-\left(\begin{array}{c}
2 m \\
n-2
\end{array}\right)+\sum_{i=1}^{\left\lfloor\frac{n+1}{2 p}\right\rfloor}\left(\begin{array}{c}
2 m+1 \\
n-2 i p+1
\end{array}\right)-\sum_{i=1}^{\left\lfloor\frac{n-1}{2 p}\right\rfloor}\left(\begin{array}{c}
2 m+1 \\
n-2 i p-1
\end{array}\right),
\end{aligned}
$$

which establishes the theorem. So it remains to prove (2). Let

$$
\left\{a_{1}, \ldots, a_{m}, b_{1}, \ldots, b_{m}, c\right\} \subset \mathfrak{h}_{m}^{*}
$$

denote the dual basis of $\left\{x_{1}, \ldots, x_{m}, y_{1}, \ldots, y_{m}, z\right\}$, let $A_{m}^{i}=\Lambda^{i}\left\langle a_{1}, \ldots, a_{m}, b_{1}, \ldots\right.$, $\left.b_{m}\right\rangle$ for $0 \leq i \leq 2 m$, let $A_{m}^{i}=0$ for all other $i \in \mathbb{Z}$, and set $A_{m}=\bigoplus_{i} A_{m}^{i}$. 
Note that $\Lambda \mathfrak{h}_{m}^{*}=A_{m} \oplus c A_{m}$, the differential $d$ is zero on $A_{m}$ and $d c=\sum_{i=1}^{m} a_{i} b_{i}$. Denote $d c$ by $\Omega_{m}$ and consider the map $\varphi_{i, m, k}: A_{m}^{i} \rightarrow A_{m}^{i+2} ; \alpha \rightarrow \alpha \Omega_{m}^{k}$. Thus $Z^{n}=A_{m}^{n} \oplus c \cdot \operatorname{ker}\left(\varphi_{n-1, m, 1}\right)$. Write $K_{n, m}=\operatorname{dim} \operatorname{ker}\left(\varphi_{n, m, 1}\right)$. Since $A_{m}^{n}$ has dimension $\left(\begin{array}{c}2 m \\ n\end{array}\right)$, we have

$$
\operatorname{dim} Z^{n}=\left(\begin{array}{c}
2 m \\
n
\end{array}\right)+K_{n-1, m}
$$

for all $n \leq m$, and in order to prove (2), it remains to show that

$$
K_{n-1, m}=\sum_{i=1}^{\left\lfloor\frac{n+1}{2 p}\right\rfloor}\left(\begin{array}{c}
2 m \\
n-2 i p+1
\end{array}\right)-\sum_{i=1}^{\left\lfloor\frac{n-1}{2 p}\right\rfloor}\left(\begin{array}{c}
2 m \\
n-2 i p-1
\end{array}\right),
$$

for all $n \leq m$, or equivalently,

$$
K_{n, m}=\sum_{i=1}^{\left\lfloor\frac{n+2}{2 p}\right\rfloor}\left(\begin{array}{c}
2 m \\
n-2 i p+2
\end{array}\right)-\sum_{i=1}^{\left\lfloor\frac{n}{2 p}\right\rfloor}\left(\begin{array}{c}
2 m \\
n-2 i p
\end{array}\right),
$$

for all $n<m$. We establish this by induction on $n$. First, notice that in characteristic $p$ one has $\Omega_{m}^{p}=0$. The following may be regarded as a finite characteristic version of the weak Lefschetz property:

Lemma. Let $0 \leq k \leq p-1, i \leq m-k, \alpha \in A_{m}^{i}$, and suppose that $\alpha \Omega_{m}^{k}=0$. Then $\alpha=\beta \Omega_{m}^{p-k}$ for some $\beta \in A_{m}^{i-2 p+2 k}$.

Proof of the lemma. First note that the result is trivial for $k=0$, so we assume $k \geq 1$. The proof is by induction on $m$. The result is obvious for $m=1$, so assume $m>1$. First notice that $\Omega_{m}=\Omega_{m-1}+a_{m} b_{m}$ and so by the binomial formula,

$$
\Omega_{m}^{j}=\left(\Omega_{m-1}+a_{m} b_{m}\right)^{j}=\Omega_{m-1}^{j}+j \Omega_{m-1}^{j-1} a_{m} b_{m},
$$

for all $1 \leq j \leq p-1$. In particular, multiplying by $a_{m}$ and $b_{m}$, respectively, gives

$$
\Omega_{m}^{j} a_{m}=\Omega_{m-1}^{j} a_{m} \quad \text { and } \quad \Omega_{m}^{j} b_{m}=\Omega_{m-1}^{j} b_{m} .
$$

Now we can write

$$
\alpha=\alpha_{0}+\alpha_{1} a_{m}+\alpha_{2} b_{m}+\alpha_{3} a_{m} b_{m},
$$

for some $\alpha_{0}, \ldots, \alpha_{3} \in A_{m-1}$. Thus $\alpha \Omega_{m}^{k}=0$ gives

$$
\alpha_{0} \Omega_{m-1}^{k}+\alpha_{1} \Omega_{m-1}^{k} a_{m}+\alpha_{2} \Omega_{m-1}^{k} b_{m}+\left(\alpha_{3} \Omega_{m-1}^{k}+k \alpha_{0} \Omega_{m-1}^{k-1}\right) a_{m} b_{m}=0,
$$

and equating coefficients, $\alpha_{0} \Omega_{m-1}^{k}=\alpha_{1} \Omega_{m-1}^{k}=\alpha_{2} \Omega_{m-1}^{k}=0$, and

$$
\alpha_{3} \Omega_{m-1}^{k}+k \alpha_{0} \Omega_{m-1}^{k-1}=0 .
$$

Since $\alpha_{1}, \alpha_{2}$ have degree 1 less than the degree of $\alpha$, their degree is $\leq(m-1)-k$, and we can apply the inductive hypothesis, which gives $\alpha_{1}=\beta_{1} \Omega_{m-1}^{p-k}$ and $\alpha_{2}=$ $\beta_{2} \Omega_{m-1}^{p-k}$, for some $\beta_{1}, \beta_{2} \in A_{m-1}^{i-2 p+2 k-1}$. Furthermore, multiplying (8) by $\Omega_{m-1}$ gives $\alpha_{3} \Omega_{m-1}^{k+1}=0$. Since $\alpha_{3}$ has degree 2 less than the degree of $\alpha$, its degree is $\leq(m-1)-(k+1)$, and we can apply the inductive hypothesis, which gives $\alpha_{3}=\beta_{3} \Omega_{m-1}^{p-k-1}$, for some $\beta_{3} \in A_{m-1}^{i-2 p+2 k}$. Let $\gamma=k^{-1} \alpha_{3} \Omega_{m-1}+\alpha_{0} \in A_{m-1}^{i}$. By (8), $\gamma \Omega_{m-1}^{k-1}=0$. Since $\operatorname{deg}(\gamma)=\operatorname{deg}\left(\alpha_{0}\right) \leq(m-1)+(k-1)$, the inductive hypothesis gives $\gamma=\eta \Omega_{m-1}^{p-k+1}$ for some $\eta \in A_{m-1}^{i-2 p+2 k-2}$. So

$$
\alpha_{0}=\gamma-k^{-1} \alpha_{3} \Omega_{m-1}=\eta \Omega_{m-1}^{p-k+1}-k^{-1} \alpha_{3} \Omega_{m-1}=\eta \Omega_{m-1}^{p-k+1}-k^{-1} \beta_{3} \Omega_{m-1}^{p-k} .
$$


Using (6),

$$
\begin{aligned}
\alpha_{0}+\alpha_{3} a_{m} b_{m} & =\left(\eta \Omega_{m-1}^{p-k+1}-k^{-1} \beta_{3} \Omega_{m-1}^{p-k}\right)+\beta_{3} \Omega_{m-1}^{p-k-1} a_{m} b_{m} \\
& =\eta \Omega_{m-1}^{p-k+1}-\beta_{3} k^{-1}\left(\Omega_{m-1}^{p-k}-k \Omega_{m-1}^{p-k} a_{m} b_{m}\right) \\
& =\eta\left(\Omega_{m}^{p-k+1}-(p-k+1) \Omega_{m}^{p-k} a_{m} b_{m}\right)-\beta_{3} k^{-1}\left(\Omega_{m-1}^{p-k}-k \Omega_{m-1}^{p-k-1} a_{m} b_{m}\right) .
\end{aligned}
$$

Hence, as we are working in characteristic $p$,

$$
\begin{aligned}
\alpha_{0}+\alpha_{3} a_{m} b_{m} & =\eta\left(\Omega_{m}^{p-k+1}+(k-1) \Omega_{m}^{p-k} a_{m} b_{m}\right)-\beta_{3} k^{-1}\left(\Omega_{m-1}^{p-k}+(p-k) \Omega_{m-1}^{p-k-1} a_{m} b_{m}\right) \\
& =\eta\left(\Omega_{m}^{p-k+1}+(k-1) \Omega_{m}^{p-k} a_{m} b_{m}\right)-\beta_{3} k^{-1} \Omega_{m}^{p-k} \quad(\text { from }(6)) \\
& =\left(\eta \Omega_{m}+(k-1) \eta a_{m} b_{m}-\beta_{3} k^{-1}\right) \Omega_{m}^{p-k} .
\end{aligned}
$$

Thus from (7),

$$
\alpha=\left(\eta \Omega_{m}+(k-1) \eta a_{m} b_{m}-\beta_{3} k^{-1}+\beta_{1} a_{m}+\beta_{2} b_{m}\right) \Omega_{m}^{p-k} .
$$

This establishes the lemma.

Returning to the theorem, the lemma gives

$$
\begin{aligned}
K_{n, m}=\operatorname{dim} \operatorname{ker}\left(\varphi_{n, m, 1}\right) & =\operatorname{dimim}\left(\varphi_{n-2 p+2, m, p-1}\right) \\
& =\left(\begin{array}{c}
2 m \\
n-2 p+2
\end{array}\right)-\operatorname{dim} \operatorname{ker}\left(\varphi_{n-2 p+2, m, p-1}\right) \\
& =\left(\begin{array}{c}
2 m \\
n-2 p+2
\end{array}\right)-\operatorname{dim} \operatorname{im}\left(\varphi_{n-2 p, m, 1}\right) \\
& =\left(\begin{array}{c}
2 m \\
n-2 p+2
\end{array}\right)-\left(\begin{array}{c}
2 m \\
n-2 p
\end{array}\right)+\operatorname{dim} \operatorname{ker}\left(\varphi_{n-2 p, m, 1}\right) \\
& =\left(\begin{array}{c}
2 m \\
n-2 p+2
\end{array}\right)-\left(\begin{array}{c}
2 m \\
n-2 p
\end{array}\right)+K_{n-2 p, m} .
\end{aligned}
$$

Thus (5) follows by induction on $n$. This completes the proof of the theorem.

Proof of Corollary 2. We maintain the notation and terminology of the proof of the theorem. For $p=2$, (5) gives

$$
K_{n, m}=\sum_{i=1}^{\left\lfloor\frac{n+2}{4}\right\rfloor}\left(\begin{array}{c}
2 m \\
n-4 i+2
\end{array}\right)-\sum_{i=1}^{\left\lfloor\frac{n}{4}\right\rfloor}\left(\begin{array}{c}
2 m \\
n-4 i
\end{array}\right)=\sum_{i=1}^{\left\lfloor\frac{n}{2}\right\rfloor}(-1)^{i+1}\left(\begin{array}{c}
2 m \\
n-2 i
\end{array}\right) .
$$

Using (3) twice gives

$$
\left(\begin{array}{c}
k \\
i
\end{array}\right)=\left(\begin{array}{c}
k-2 \\
i
\end{array}\right)+2\left(\begin{array}{c}
k-2 \\
i-1
\end{array}\right)+\left(\begin{array}{c}
k-2 \\
i-2
\end{array}\right) .
$$

Thus

$$
K_{n, m}=\left(\begin{array}{c}
2 m-2 \\
n-2
\end{array}\right)+2 \sum_{i=1}^{\left\lfloor\frac{n-1}{2}\right\rfloor}(-1)^{i+1}\left(\begin{array}{c}
2 m-2 \\
n-1-2 i
\end{array}\right)
$$

Hence

$$
K_{n, m}=\left(\begin{array}{c}
2 m-2 \\
n-2
\end{array}\right)+2 K_{n-1, m-1}
$$

for all $n<m$. Let $\Delta_{n, m}=\operatorname{dim} H^{n}\left(\mathfrak{h}_{m}\right)-\operatorname{dim} H^{n-1}\left(\mathfrak{h}_{m}\right)$. We will show that $\Delta_{n, m}>0$ for all $1 \leq n \leq m$. The proof is by induction on $n+m$. First notice that 
since $\operatorname{dim} H^{1}\left(\mathfrak{h}_{n}\right)=2 n$ and $\operatorname{dim} H^{0}\left(\mathfrak{h}_{n}\right)=1$, one has $\Delta_{1, n}>0$ for all $n \geq 1$. From (11), (3) and (44), for all $n \leq m$,

$$
\Delta_{n, m}=\left(\begin{array}{c}
2 m \\
n
\end{array}\right)-\left(\begin{array}{c}
2 m \\
n-1
\end{array}\right)-\left(\begin{array}{c}
2 m \\
n-2
\end{array}\right)+\left(\begin{array}{c}
2 m \\
n-3
\end{array}\right)+K_{n-1, m}-K_{n-3, m} .
$$

So, employing (9) and (10) gives

$$
\begin{aligned}
\Delta_{n, m}= & \left(\begin{array}{c}
2 m-2 \\
n
\end{array}\right)+\left(\begin{array}{c}
2 m-2 \\
n-1
\end{array}\right)-2\left(\begin{array}{c}
2 m-2 \\
n-2
\end{array}\right)-\left(\begin{array}{c}
2 m-2 \\
n-3
\end{array}\right)+\left(\begin{array}{c}
2 m-2 \\
n-4
\end{array}\right) \\
& +2 K_{n-2, m-1}-2 K_{n-4, m-1} \\
= & \left(\begin{array}{c}
2 m-2 \\
n
\end{array}\right)-\left(\begin{array}{c}
2 m-2 \\
n-1
\end{array}\right)+\left(\begin{array}{c}
2 m-2 \\
n-3
\end{array}\right)-\left(\begin{array}{c}
2 m-2 \\
n-4
\end{array}\right)+2 \Delta_{n-1, m-1} .
\end{aligned}
$$

The inductive hypothesis gives $\Delta_{n-1, m-1}>0$. Thus, since $\left(\begin{array}{c}2 m-2 \\ n\end{array}\right) \geq\left(\begin{array}{c}2 m-2 \\ n-1\end{array}\right)$ and $\left(\begin{array}{c}2 m-2 \\ n-3\end{array}\right) \geq\left(\begin{array}{c}2 m-2 \\ n-4\end{array}\right)$ for all $n \leq m$, we have $\Delta_{n, m}>0$, as required.

Remark. The characteristic zero result of [4 can be deduced from the above theorem by choosing a sufficiently large prime $p$. Indeed, the Heisenberg algebras are defined over $\mathbb{Z}$, and in each dimension, the determination of the cohomology amounts to the computation of the rank of an integer matrix representing the differential. But for an integer matrix, the rank in characteristic zero can only differ from the rank in characteristic $p$ for finitely many values of $p$.

\section{REFERENCES}

1. Grant F. Armstrong, Unimodal Betti numbers for a class of nilpotent Lie algebras, Comm. Algebra 25 (1997), no. 6, 1893-1915. MR1446138 (98e:17031)

2. Grant F. Armstrong, Grant Cairns, and Barry Jessup, Explicit Betti numbers for a family of nilpotent Lie algebras, Proc. Amer. Math. Soc. 125 (1997), no. 2, 381-385. MR 1353371 (97d:17013)

3. Hannes Pouseele, On the cohomology of extensions by a Heisenberg Lie algebra, Bull. Austral. Math. Soc. 71 (2005), no. 3, 459-470. MR2150935 (2006c:17028)

4. L. J. Santharoubane, Cohomology of Heisenberg Lie algebras, Proc. Amer. Math. Soc. 87 (1983), no. 1, 23-28. MR677223 (84b:17010)

5. Emil Sköldberg, The homology of Heisenberg Lie algebras over fields of characteristic two, Math. Proc. R. Ir. Acad. 105A (2005), no. 2, 47-49 (electronic). MR2164586

Department of Mathematics, La Trobe University, Melbourne, Australia 3086

E-mail address: G.Cairns@latrobe.edu.au

Department of Mathematics, La Trobe University, Melbourne, Australia 3086

E-mail address: Sebastian@momo.math.rwth-aachen.de 